\title{
ESCRITOS JÓVENES
}

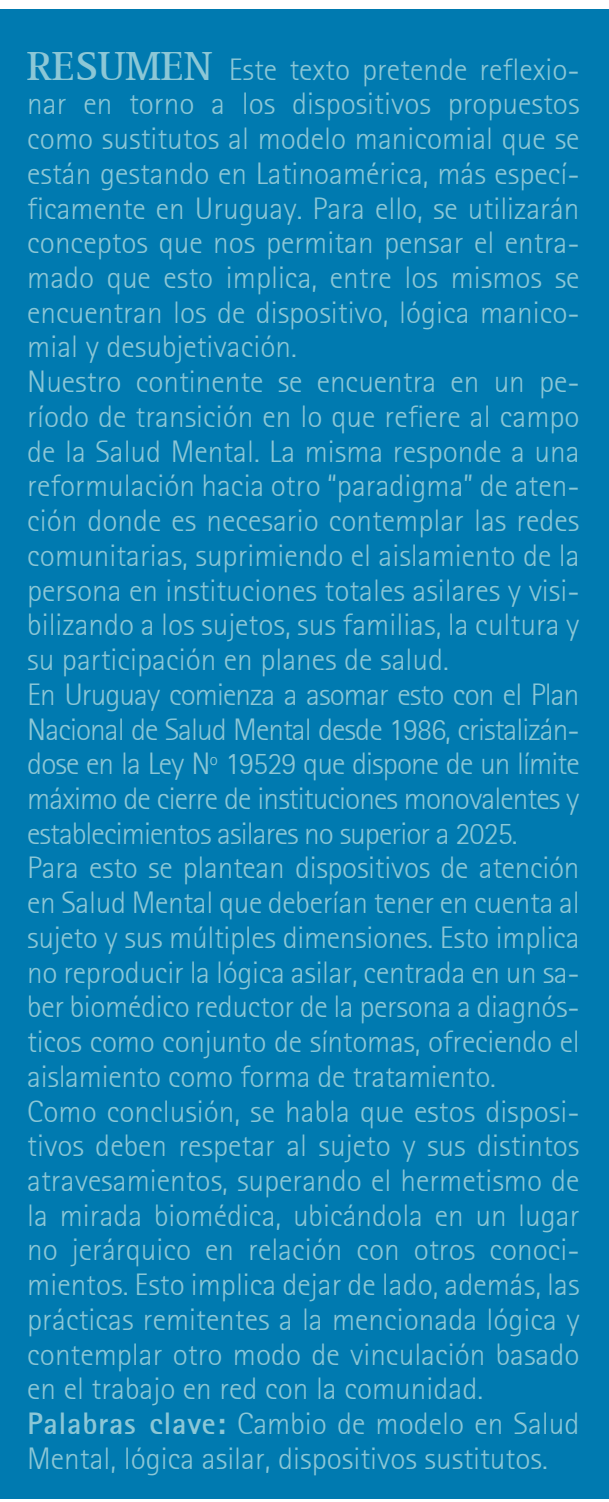

${ }^{1}$ Artículo recibido el 30 de mayo de 2019. Aprobado el 3 de agosto de 2020.

${ }^{2}$ Licenciado en Psicología; Maestrando en Psicología Social- Facultad de Psicología- UdelaR. Trabajador de dispositivo sustituto al modelo asilar.Mail: sc.juanpi@ gmail.com

\section{INTRODUCCIÓN}

Para entender la problemática que implica la creación de dispositivos sustitutivos al manicomio, podemos distinguir a grandes rasgos dos períodos del modelo asilar en Europa. El primero, desde 1880 hasta 1950, denominado como del "auge del asilo" (Desjarls, cito en Thornicroft y Tansella, 2003), donde el manicomio es la principal estructura que se encarga del cuidado y de mantener las necesidades básicas del sujeto. Es el "tratamiento" por excelencia de la época, oficiando como custodia del sujeto y aislándolo de su contexto social.

A partir de 1950, esta institución comienza a ser cuestionada, entre otras cosas, por los impactos negativos que genera en las personas internadas como en los trabajadores. Esto da inicio a un segundo momento que se conoce como el "declive del asilo" (Wing y Brown, cito en Thornicroft y Tansella, 2003). En este período surgen planteamientos en torno a los procesos de institucionalización (Goffman, [1970]1994) de las personas internadas por un tiempo prolongado, en donde la adaptación a la rutina y dinámica institucional genera, entre otras cosas, la pérdida de habilidades sociales, así como un proceso de desculturización (Aguirre y Rodríguez, 1995) dado por el aislamiento.

Alrededor de 1970, en Europa comienzan a surgir experiencias y cuestionamientos que giran sobre la necesidad de crear formas inclusivas, justas y humanas de trato entre las personas y abolir el encierro (Basaglia, 2008; Amarante, 2009; Dell Aqua, s.f.; Greber, 2013). Estas experiencias influyen en diversos paises del cono sur, inspirando a otros movimientos de reforma, como ejemplo el de Brasil (Amarante et al., s.f.) ${ }^{3}$.

Como resultado de la influencia que estos planteos tuvieron a nivel internacional se plantea la necesidad, por parte de diversos organismos internacionales, de una reformulación de la atención en Salud Mental (SM) con miras a la creación de otro "paradigma". Este estará centrado en dar respuesta a partir del diseño de abordajes comunitarios y ya no en el aislamiento social del sujeto en estas instituciones. A su vez, se propenderá a que las personas participen en lo que tiene que ver con sus propios pro- 
De Prácticas y discursos

Universidad Nacional del Nordeste

Centro de Estudios Sociales

Año 9, Número 14, 2020, Octubre

ISSN 2250-6942

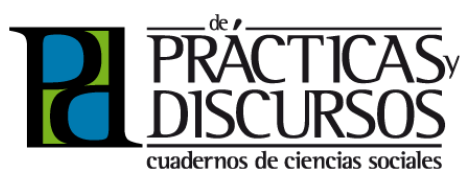

ABSTRACT This text aims to reflect on the devices proposed as substitutes for the asylum model that is brewing within Latin America, more specifically in Uruguay. For this, concepts that allow us to think about the framework that this implies will be used, among them are that of device, asylum logic and desubjectivity.

Our continent is in a period of transition regar-

ding the field of Mental Health. It responds to a reformulation towards another "paradigm" of care where it is necessary to contemplate community networks, suppressing the isolation of the person in total asylum institutions. Making the subjects, their families, the culture and their participation in health plans visible.

Within Uruguay, this begins to emerge with the National Mental Health Plan of 1986, crystallizing in the Law No. 19,529 that has a maximum closure limit for monovalent institutions and asylum establishments not exceeding 2025.

For this, care devices are proposed in Mental Health that should take into account the subject and its multiple dimensions. This implies not reproducing the asylar logic, centered on a biomedical knowledge that reduces the person to diagnoses as a set of symptoms, offering isolation as a form of treatment.

In conclusion, it is said that these devices must respect the subject and its different edges, overcoming the hermeticism of the biomedical gaze placing it in a non-hierarchical place in relation to other knowledge. This implies leaving aside the practices that refer to the aforementioned logic and contemplating another way of Relationship based on networking with the community.

Keywords: Model change in Mental Health, asylar logic, substitute devices. cesos de salud mediante la elaboración de políticas y planes de salud, entre otros (OPS-OMS, 1990, 2005, 2010, 2013).

En Uruguay, el primer Plan Nacional de Salud Mental (PNSM) aprobado data de $1986^{4}$, donde por primera vez se propone una reestructura de la atención en SM, la cual, entre otras cosas, genera que el Hospital Nacional Psiquiátrico pase a ser un hospital de agudos y las Colonias, para pacientes crónicos. Por otra parte, se generan políticas de descentralización de la atención (creación de policlínicas y centros diurnos barriales) y desinstitucionalización que, por razones políticas y presupuestales, quedan truncas hasta 2010 (Baroni, 2015). En ese año, las organizaciones sociales comienzan a impulsar el debate sobre un cambio de modelo que conlleva un cambio legislativo en el Uruguay, ya que la ley vigente databa de 1936. En 2017, se aprueba la Ley de Salud Mental № 19529, la cual incluye, entre otras cosas, al 2025 como fecha de cierre para las instituciones monovalentes y asilares.

Este cambio jurídico y el compromiso de cerrar estas instituciones acelera la necesidad de crear soluciones habitacionales para aquellas personas que han vivido por años en las mismas. La Ley 19529, en su reglamentación (ordenanza № 1046), si bien define cómo serán algunos de los dispositivos sustitutos al modelo asilar, paradojalmente no deja claro qué personal estará a cargo de los mismos, lo cual anima las siguientes preguntas: ¿Cómo será el trato hacia los sujetos que se encuentren viviendo en ellos? ¿Se reproducirá la lógica asilar como forma de vinculación? ¿Se generará otra lógica de relacionamiento que responda a un modelo psicosocial, evitando así la reproducción del modelo que se pretende eliminar?

\section{DEL ASILO Y SU LÓGICA}

Primeramente, resulta necesario resaltar que las instituciones asilares son anteriores al tratamiento psiquiátrico. Foucault ([1968]1998) nos menciona que el cometido de los hospicios, anteriores al siglo XIX, era el de aislar y encerrar, no el de "tratar". Con el advenimiento del positivismo, comienza a darse un cambio de paradigma que deriva poco a poco en la conquista de estos espacios por parte de la medicina y la psiquiatría. De ver al "loco" como una persona poseída (Edad Media) o persona ociosa (época clásica), se la comienza a categorizar como "enfermo mental" y a implementar la idea de tratamiento y rehabilitación. Sin embargo, el aislamiento y encierro perduran 
como formas de abordar el problema de "la cura".

Una de las particularidades de las instituciones totales (Goffman, [1970] 1994), como consecuencia de transitar internaciones prolongadas y monótonas dentro de una única estructura donde, en caso de que no haya visitas, no se interactúa con otras personas que no sean internados o trabajadores, es la de hundir al sujeto en el olvido y el aislamiento.

Por ello, la institución manicomial produce una nueva subjetividad que, basada en el encierro, reemplaza todas las dimensiones de la vida del individuo, el cual se adecua a la situación de aislamiento (Galende, 2008). Debido a esto, la institución misma altera el orden social básico (Goffman,[1970] 1994), siendo la única opción para vivir que se le impone al sujeto.

Por otra parte, en la institución manicomial aún prima el abordaje desde el modelo médico hegemónico que contempla al sujeto como un ente puramente biológico, excluyendo su parte histórica y social. Además, esto devela una relación asimétrica entre profesional y "paciente", no teniéndose en cuenta a este último como ser histórico contextual (Menéndez, 2009).

A su vez, estas estructuras operan bajo una lógica denominada manicomial que resulta de un conjunto de conceptos y prácticas que direccionan las relaciones trabajador-usuario dentro y fuera de la institución (Ferreira de Oliveira, 2009). Sumado a la visión que tienen del sujeto como objeto, el pasar a ser la persona tratada, según su diagnóstico, le otorga pero también le sustrae derechos. De igual modo, si a la persona se la califica como persona improductiva, peligrosa e incurable, se acarrea con procesos de exclusión, dependencia y tutelaje indefinido, perdiendo así autonomía, ya que para esta lógica es imposible curarse.

Es menester, entonces, considerar que desde esa concepción se desconoce al sujeto como tal, a la vez que se promueve un (auto)estigma social donde predomina el diagnóstico y una forma impuesta de tratamiento donde la persona no tiene voz o voto y se dejan de lado las potencialidades del ser humano, asi como el saber que se lleva consigo (Correa-Urquiza y Martínez, 2017; Baroni, 2018).

Cabe entonces preguntarse nuevamente, ante el cierre de estas instituciones y un supuesto cambio hacia un paradigma psicosocial, con la apertura de dispositivos que sustituyan al manicomio, ¿se seguirá reproduciendo esta lógica? 
De Prácticas y discursos

Universidad Nacional del Nordeste

Centro de Estudios Sociales

Año 9, Número 14, 2020, Octubre

ISSN 2250-6942

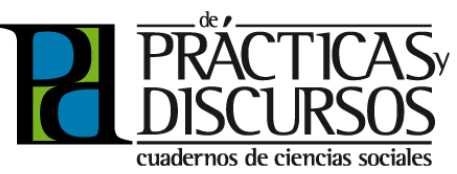

cuadernos de ciencias sociales

\section{DE LOS DISPOSITIVOS, SU PLANTEAMIENTO Y EL TRATO}

Foucault introduce la noción de dispositivo como una forma de poder ver un conjunto de elementos heterogéneos, discursivos o no (prácticas, instituciones, reglamentos, enunciados científicos, etcétera), con el objetivo de comprender las relaciones de poder y saber que se generan.

Para Heredia (2012), además, los dispositivos surgen como una respuesta política a un problema de orden práctico e histórico. Por lo tanto, se plantean estas formaciones como respuestas a una "función estratégica dominante", donde entra en juego la invención de procedimientos y técnicas de poder.

para producir e inducir en una multiplicidad de individuos determinadas formas de conducta y habilitan, con su eficacia, a un contagio y una re-territorialización en diversas instituciones, así como a un perpetuo proceso de reactualización funcional [...] su génesis se da en una relación concreta de fuerzas y, específicamente, en una relación disimétrica en la cual uno de los polos logra producir efectos sobre el otro. (Heredia, 2012: 89)

En el caso particular uruguayo, la necesidad de cerrar para 2025 las instituciones asilares y monovalentes obliga a diseñar dispositivos alternativos.

Teniendo en cuenta la definición traída por el autor, y pensando que los dispositivos sustitutivos al manicomio van a estar relacionados con un sistema de $\mathrm{SM}$ inserto en un sistema de salud con dominación del saber biomédico (Correa-Urquiza y Di Giacomo, 2013), es que surge nuevamente el interrogante sobre si se seguirá reproduciendo la lógica asilar en estos dispositivos. Esta pregunta resuena más cuando los autores proponen que ese saber se vincula de forma hegemónica con otros conocimientos, anexándolos asi (en el caso de otras disciplinas) o minimizando el saber proveniente del sujeto (colonizando su identidad y reduciéndolo a un diagnóstico) dado su carácter de saber profano. De hecho, Costa-Rosa (2000) establece que, si bien se ha buscado generar dispositivos "extra-hospitalares" dentro del modelo asilar, al ser la institución hospital psiquiátrico la regente en ese paradigma, se terminan reproduciendo los mismos parámetros. Esto hace que nuestra pregunta resuene más ampliamente.

Pensamos que una forma de evitar lo anterior sea que las 


\footnotetext{
${ }^{5}$ La condición de persona es comprendida por la autora como un status adjudicado y reconocido por otros dentro de un contexto de relacionamiento mediante vínculos sociales y de bienestar.
}

propuestas sustitutivas al manicomio no se posicionen solamente desde una lógica hegemónica biologicista, lo que implica una constante vigilancia de las prácticas a realizarse, además de generar espacios de formación sobre la base de los Derechos Humanos, de lo contrario se estará reproduciendo el modelo que pretenden superar.

De hecho, el cerrar los manicomios, dificilmente impida que las lógicas manicomiales se produzcan en otros sitios institucionales (Baroni, 2015). Sin embargo, el cierre de las instituciones asilares y monovalentes abrirá un nuevo impulso para crear experiencias que potencien nuevas formas vinculares basadas en los derechos de los sujetos. Esto permitirá terminar con la ecuación locura-encierro y así combatir el estigma de la doble exclusión (pobre y loco).

Viendo esta situación, es necesario repensar la noción de dispositivo, el cual posee una forma de visualizar aspectos de la realidad y su entramado en las relaciones de poder-saber-sujeto. Si los dispositivos "son máquinas para hacer ver y hablar" (Deleuze, 1990: 155), entonces, cada dispositivo nos permitirá ver en qué concepciones se sustenta (lo que enuncia) y cómo va construyendo las mismas (lo mutable y sus derivas). Los dispositivos tienen diversas líneas de composición que permiten vislumbrar las relaciones de poder, así como producen nuevas subjetividades y/o reproducen las existentes. Ellos, por lo tanto, nos permiten seguir también las líneas de subjetivación y las líneas de fuga que generan caminos creativos, abriendo paso a lo nuevo, lo novedoso.

Lo interesante aqui es que lo que se produzca en los dispositivos sustitutivos al manicomio no tiene por qué repetir el modelo asilar y, para ello, un paso importante es no situar jerárquicamente al modelo médico, entre otras cosas, porque desde alli se reproduce la idea de enfermedad basado en un ideal de la cura, con limitaciones teóricas y tecnológicas, que generan frustración y pesimismo en las personas y sus allegados (Pérez, 2016). Por otra parte, el proceso de subjetivación del "paciente" permite que haya más "enfermos" como una totalidad y menos sujetos con la complejidad que ello implica.

Otro aspecto fundamental que aqui se desprende es que estos dispositivos sustitutivos deben reconocer la condición de persona ${ }^{5}$ de los sujetos (Beuniak, cito en Pérez, 2016). De lo contrario, y a partir de procesos de desubjetivación, el sujeto pierde su categoría como tal, solo quedando la posibilidad de ser desde la enfermedad, lo que implica, en muchos casos, perder hasta la capacidad de poder decidir (Pérez, 2016).

\section{| PAGINA 05 | ESCRITOS JÓVENES |}


De Prácticas y discursos

Universidad Nacional del Nordeste

Centro de Estudios Sociales

Año 9, Número 14, 2020, Octubre

ISSN 2250-6942

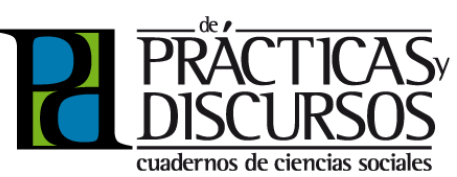

${ }^{6}$ Agamben (1998) trabaja este concepto basándose en el planteamiento aristotélico de animal político (zoon politikon), la Nuda Vida responderá a que en la vida política a la persona se le sustraigan sus derechos y la calidad de ciudadano, deviniendo un Zoon (animal).
Por otra parte, para romper con el encierro estructural y mental de la comunidad, pensamos que estos dispositivos deben trabajar insertos en una red territorial. Tavares de Assis et al (2014) menciona que esta labor tiene como objetivo superar el aislamiento de las prácticas del servicio de salud y poner dentro del territorio social la posibilidad de creación en conjunto (personas-contexto) de nuevas formas de relacionarse con proyectos comunes. Formar redes implica, entonces, promover una acción coordinada para que un devenir común se organice aportando a un cambio de imaginario sobre esta problemática.

Pensar en territorio, el cual es definido como un espacio donde se despliegan relaciones, experiencias y circulan los sujetos construyendo instancias personales e institucionales, como un espacio psicosocial (Tavares de Assis et al., 2014), donde la articulación sujetos-redes-territorio-comunidad permitirá operar superando la Nuda Vida ${ }^{6}$ (Agamben, 1998). Esto implica que las personas participen de la comunidad (polis), circulen por el territorio y que puedan ser parte de proyectos comunes. Considerar lo anterior en conjunto con un modelo de red y de base comunitaria en salud, implica trascender el viejo concepto de enfermedad mental y su tratamiento (Amarante, s.f.) Proponer alternativas a esta forma de pensamiento significa poner a la enfermedad dentro de un paréntesis (Basaglia, cito en Amarante et al., s.f.) para acercarse a los sujetos intentando comprender por qué sufren, cuáles son sus necesidades y cómo podemos resolverlas, construyendo proyectos en común que habiliten la expresión de la diversidad.

Finalizando este apartado, se piensa que los dispositivos sustitutos deben adoptar, para ser alternativos, una forma distinta de pensar a las personas. Esto implica el evitar aprisionarlas en una categoría y respetar la multiplicidad de cada ser humano. El camino para lograrlo quizás sea, como lo mencionan algunos autores, con una fuerte vigilancia práctica y teórica que tenga base en Derechos Humanos, habilitando a la persona a desarrollar sus potencialidades.

\section{CONCLUSIONES}

Considerando los conceptos tratados, es que se interroga desde qué lugar se situarán las prácticas de los dispositivos sustitutivos al manicomio para no generar Nuda Vida.

Vimos cómo el aislamiento social y la reducción del sujeto a un diagnóstico psiquiátrico pueden seguir operando tanto a nivel 
institucional como social.

Por otra parte, como ya se dijo, un dispositivo permite realizar una analítica de las relaciones de poder $y$, en este caso, implica también pensar y resituar el saber médico como conocimiento imperante. Planteamos que las propuestas sustitutivas al modelo asilar deberán situar a ese corpus conceptual en un lugar horizontal con relación a otros saberes. Esto posibilitará una mirada compleja del sujeto, habilitando voz y voto a la hora de decidir su vida; lo que conlleva asimismo a dar lugar a creaciones noveles, singulares, ante las propuestas clásicas de "tratamiento".

Surgen asi múltiples interrogantes que carecen de respuesta aún. Entre ellas, si los dispositivos sustitutivos se instalan, ¿cómo se realizará la reestructura de las instituciones asilares? ¿Se seguirá reproduciendo un saber que contemple solo una arista del sujeto quedando anclado a un modelo biomédico?

Con relación al trabajo en los dispositivos sustitutivos, ¿se podrá realizar el trabajo en red territorial y comunitaria? En consecuencia, ¿en estos dispositivos tendrán lugar prácticas donde se tomen en cuenta el saber del sujeto, su deseo y su existencia social?

Lo cierto aqui es que nada impide que se reproduzca dentro de estos espacios la lógica manicomial, reviviendo otra vez el modelo institucional al cual remite. Como ya se mencionó, para que esto no ocurra es necesario una vigilancia constante de base en Derechos Humanos, además de un trabajo en conexión con el territorio y la comunidad. Es fundamental plantear que al encierro lo rompe la red y que el sujeto se construye con un otro social que le devuelva su cualidad de persona. Existe una esperanza, entonces que los dispositivos funcionen como habilitantes a una producción de nuevas subjetividades que hagan luz en la persona y el contexto. Implica igualmente un compromiso por parte de los trabajadores y la comunidad para que el ser humano pueda desarrollar sus potencialidades, logrando una verdadera autonomía.

\section{BIBLIOGRAFIA}

AGAMBEN, G. (2006). Homo sacer. El poder soberano y la nuda vida. Valencia: Pre-textos.

AGUIRRE, A. Y RODRíGUEZ, A. (1995). Patios abiertos y patios cerrados. Psicología cultural de las organizaciones. Barcelona: Marcombo. 
AMARANTE, P. (2009). Superar el manicomio: salud mental y atención psicosocial. Buenos Aires: Topía.

AMARANTE, P.; BARROS DE OLIVEIRA, M.; TORRES, E. Y COHELO, I. (S.F.) Derechos Humanos y Salud Mental en Brasil: una historia de lucha y militancia por la justicia social y el derecho a la vida-"la salud no se vende, la locura no se encierra". Disponible en http://www.atopos.es/images/eatopos2/4.pdf

ASAMBLEA INSTITUYENTE, POR SALUD MENTAL, DESMANICOMIALIZACIÓN Y VIDA DIGNA (2017, OCTUBRE). Comunicado a la opinión pública. Recuperado de: http://asambleainstituyente.blogspot.com/

BARONI, C. (2015). Perspectivas y proyecciones de la Salud Mental en el Uruguay. En SERPAJ. Derechos Humanos en el Uruguay. Informe Anual. Montevideo: Tradinco. ISBN: 978-9974-564-41-1

(2018). Para abrir hay que cerrar. Zur, Pueblo de voces. Recuperado de: https://www.academia.edu/36604123/Para_ abrir_hay_que_cerrar..._Apuntes_acerca_de_la_Ley_de_Salud_mental_en_Uruguay

BASAGLIA, F. (2008). La condena de ser pobre y loco: alternativas al manicomio. Buenos Aires: Topía.

COSTA-ROSA, A. (2000). "O modo psicossocial: um paradigma das práticas substitutivas ao modo asilar". En Amarante, P. (ed.) Ensaios: subjetividade, saúde mental, sociedade (pp. 141168). Rio de Janeiro: Fiocruz. ISBN 978-85-7541-319-7.

CORREA-UROUIZA, M. Y MARTÍNEZ-HERNÁEZ, A. (2017). Un saber menos dado: nuevos posicionamientos en el campo de la salud mental colectiva. En Salud Colectiva, (2) 13, 267-278. [En línea]. [Accedido: 20 de febrero de 2018]. Disponible en: <https:// doi.org/10.18294/sc.2017.1168>.ISSN 1851-8265.

CORREA UROUIZA M. Y DI GIACOMO S. (2013). Radio Nikosia: Mutiny on the ship of fools. En Araoz, G.; Alves, G. y Jawroski, K. (comp.) Rethinking Madness. Interdisciplinary and Multicultural Reflections. Oxford, Reino Unido: Inter-disciplinary Press. 
DELEUZE, G. (1990). "¿Qué es un dispositivo?" En Foucault, filósofo. Buenos Aires: Gedisa.

FERREIRA DE OLIVEIRA, W. (2009). Éticas em conflito: reforma psiquiátrica e lógica manicomial. En Cadernos Brasileiros de Saúde Mental, 1(2), 42-56. Recuperado de: http://incubadora. periodicos.ufsc.br/index.php/cbsm/article/view/1126/1362

FOUCALUT, M. (1998). Historia de la locura en la época clásica. Tomo 1. Bogotá: Fondo de Cultura Económica.

GALENDE, E. (2008). Desmanicomialización institucional y subjetiva. Psicoanálisis. (30) 2/3, 395-427.

GINÉS, A.; PORCIÚNCULA, H. Y ARDUINO, M. (2005). El plan de Salud Mental: veinte años después. Evolución, perspectivas y prioridades. En Revista de psiquiatría del Uruguay, (69)2, 129-150.

GOFFMAN, E. (1994). Internados. Ensayo sobre la situación social de los enfermos mentales. Buenos Aires: Amorrortu.

GREBER, L. (2013). "CLINIQUE LA BORDE, un espacio de vida". En De León, N. (ed.) Abrazos: Experiencias y narrativas acerca de la locura y la Salud Mental (pp. 125-147). Montevideo: Levy.

HEREDIA, J. (2012). Dispositivos y/o Agenciamientos. Contrastes. Revista Internacional de Filosofia, (19)1 (2014), 83-101. Málaga, España: Departamento de Filosofía, Universidad de Málaga, Facultad de Filosofía y Letras Campus de Teatinos, E-29071. Recuperado de: https://www. uma.es/contrastes/pdfs/019/5-Juan_Manuel_Heredia.pdf. ISSN: 1136-4076

MENÉNDEZ, E. (2009). De sujetos, saberes y estructuras. Introducción al enfoque relacional en el estudio de la salud colectiva. Buenos Aires: Lugar.

PÉREZ FERNÁNDEZ, R. (2016). Las dolencias de la mente. Prácticas de atención y cuidado de personas con demencia en Uruguay. Departamento de Salud Comunitaria, Universidad Nacional de Lanús, Remedios de Escalada, República Argentina. 
TAVARES DE ASSIS, J.; BARREIROS, C.; MOREIRA, A.; TYKANORI, R.; DE LEMOS, P.; DUARTE, T.; NICÁCIO, F.; DA COSTA, M.; SIMÕES DE SOUZA, I. Y TEXEIRA, A. (2014). Política de saúde mental no novo contexto do Sistema Único de Saúde: regiões e redes. Divulgação em saúde para debate, 52, 88-113. Rio de Janeiro, OUT.

THORNICROFT, G. Y TANSELLA, M. (2003). What are the arguments for community-based mental health care. Copenhagen: WHO regional Office for europe. Recuperado de: http:// www.euro.who.int/_data/assets/pdf_file/0019/74710/E82976. pdf Documentos oficiales

MINISTERIO DE LA REPÚBLICA FEDERATIVA DE BRASIL, ORGANIZACIÓN PANAMERICANA DE LA SALUD Y ORGANIZACIÓN MUNDIAL DE LA SALUD (2005). Principios de Brasilia: Principios Rectores para el desarrollo de la atención en salud mental. Recuperado de: http://www.psi.uba.ar/academica/carrerasdegrado/ psicologia/sitios_catedras/obligatorias/066_salud2/material/normativas_legislaciones/ops_oms_principios_brasilia.pdf

ORGANIZACIÓN PANAMERICANA DE LA SALUD Y OFICINA REGIONAL DE LA ORGANIZACIÓN MUNDIAL DE LA SALUD PARA LAS AMÉRICAS (1990, 14 DE NOVIEMBRE). Declaración de Caracas. En el marco de la Conferencia Reestructuración de la Atención Psiquiátrica en América Latina, organizada. Caracas, Venezuela. Recuperado de: https://www.oas.org/dil/esp/ declaracion_de_caracas.pdf

ORGANIZACIÓN PANAMERICANA DE LA SALUD, ORGANIZACIÓN MUNDIAL DE LA SALUD, GOBIERNO DE PANAMÁ (2010, 7 Y 8 DE OCTUBRE). Consenso de Panamá. En el marco de la Conferencia Regional de Salud Mental. Recuperado de: http://www1.paho. org/hq/dmdocuments/2011/1.\%20PosterSpanishJAN11.pdf

ORGANIZACIÓN PANAMERICANA DE LA SALUD, ORGANIZACIÓN MUNDIAL DE LA SALUD, SUS, MINISTÉRIO DA SAÚDE Y GOBIERNO FEDERAL BRASIL, PAIS RICO É PAIS SEM POBREZA (2013). Consenso de Brasilia 2013. [En línea]. Recuperado de: https://www.paho.org/hq/dmdocuments/2014/ BRASILIA-CONSENSUS-2013span.pdf 
URUGUAY. PODER LEGISLATIVO (1936). Ley Psicópatas. Se organiza la asistencia. Recuperado de: https://legislativo.parlamento.gub.uy/temporales/leytemp2721368.htm

URUGUAY. MINISTERIO DE SALUD PÚBLICA. COMISIÓN NACIONAL DE SALUD MENTAL (1986). Plan Nacional de Salud Mental. Recuperado de: http://www.msp.gub.uy/programa/ plan-nacional-de-salud-mental-1986

URUGUAY. PODER LEGISLATIVO (2017). Salud Mental. Desde una perspectiva de Derechos Humanos en el marco del Sistema Nacional Integrado de Salud. Recuperado de: https://www. impo.com.uy/bases/leyes/19529-2017

URUGUAY. MINISTERIO DE SALUD PÚBLICA (2019). Ordenanza 1040. Recuperado de: https://www.gub.uy/ministeriosalud-publica/institucional/normativa/ordenanza-1046019-redatencion-salud-mental 